\title{
Direct monitoring of cancer-associated mRNAs in living cells to evaluate the therapeutic RNAi efficiency using fluorescent nanosensor
}

Seong Min Ahn, ${ }^{\dagger,+}$ Seounghun Kang, ${ }^{\dagger,+}$ and Dal-Hee Min ${ }^{* \dagger,+, \&}$

'Department of Chemistry, Seoul National University, Seoul, 08826, Republic of Korea

*Center for RNA Research, Institute for Basic Science (IBS), Seoul, 08826, Republic of Korea

${ }^{\S}$ Institute of Biotherapeutics Convergence Technology, Lemonex Inc., Seoul, 08826, Republic of Korea

\section{Experimental Section}

Materials. Graphite nanofiber was purchased from Carbon Nanomaterial Technology (Korea). Sulfuric acid $\left(\mathrm{H}_{2} \mathrm{SO}_{4}\right)$ and hydrochloric acid $(\mathrm{HCl})$ were purchased from Samchun chemical (Korea). Hydrogen peroxide $\left(\mathrm{H}_{2} \mathrm{O}_{2}\right) 30 \%$ in water were purchased from Junsei (Japan). Potassium permanganate $\left(\mathrm{KMnO}_{4}\right)$, potassium persulfate $\left(\mathrm{K}_{2} \mathrm{~S}_{2} \mathrm{O}_{8}\right)$, phosphorus pentoxide $\left(\mathrm{P}_{2} \mathrm{O}_{5}\right)$ and ammonium hydroxide $\left(\mathrm{NH}_{4} \mathrm{OH}\right) 28-30 \%$ in water were purchased from Sigma-Aldrich (USA). Phosphate buffered saline (PBS), Dulbecco's modified eagle's medium (DMEM), fetal bovine serum (FBS) and antibiotics (penicillin and streptomycin) were purchased from WelGENE (Korea). siRNA and synthetic single stranded RNA were synthesized by Bioneer (Korea). Lipofectamine ${ }^{\circledR} 2000$ was purchased from Invitrogen (USA). Primers for RT-PCR were obtained from Cosmogenetech (Korea). Fluorescent dye labeled PNAs were purchased from Panagene (Korea). PNU-74654 was purchased from Sigma-Aldrich (USA).

Synthesis and characterization of D-GON. D-GON was synthesized according to our previously published method. To investigate physicochemical changes in GON modified with dextran, absorbance spectrum was obtained by using UV-vis spectrophotometer S-3100 (Scinco, Korea). FT-IR spectrum was recorded on Vertex 
70 FT-IR spectrometer equipped with HYPERION 1000 microscope (Bruker, USA). The measurements of the average particle size and zeta potential were carried out using Zetasizer Nano S dynamic light scattering (DLS) analyzer (Malvern instrument, UK). Enhancement of stability in $1 \times$ PBS solution was monitored by the naked eye.

Cell cytotoxicity assay. Cytotoxicity of the D-GONs was evaluated by Cell Counting Kit-8 (CCK-8) toward HepG2 cells. HepG2 cells were seeded on 96-well cell culture plate and cultured with growth medium for 24 hours $\left(37^{\circ} \mathrm{C}, 5 \% \mathrm{CO}_{2}\right)$. The cells were treated with various concentrations of the as-prepared D-GONs in serum-free medium for 12 hours. Then $10 \mu \mathrm{L}$ of the CCK- 8 solution was added to each well of plate. After the plate was incubated for 2 hours, the absorbance of each well was recorded at $450 \mathrm{~nm}$ by microplate reader. Mean and standard deviation of triplicate values were calculated and plotted.

Preparation of siRNA, PNA probe and target RNA. Highly functional siRNA for targeted RNA interference was selected with online design tool provided by Dharmacon siDESIGN Center. siRNAs consist of a 19 mer double-stranded RNA with a deoxythymidine dinucleotide (dTdT) overhang at the 3' ends of each strand. PNA probe sequence is identical to the antisense strand of siRNA except dTdT overhang to specifically demonstrate siRNA-mediated cleavage of target mRNA. Also, PNA probe is labeled by Cyanine 5 (Cy5) dye at 5' end with di-ethylene glycol linker to distance the hybridization portion and improve solubility. The sequence of target RNA, single-stranded RNA, is entirely complementary to each PNA probe.

The sequence information of siRNA, PNA probe and target RNA is as follow.

- $\quad$ siRNA targeting $\beta$-catenin mRNA (5'- 3'): sense; ACA UCA AGA AGG AGC UAA A, antisense; UUU AGC UCC UUC UUG AUG U.

- $\quad$ PNA probe (5'- 3'): $\beta$-actin; ATC TTG ATC TTC ATT GTG C, $\beta$-catenin; TTT AGC TCC TTC TTG ATG T, cyclin D1; TTC TGC ACA CAT TTG AAG T, c-Myc; TTC CTC ATC TTC TTG TTC C, scrambled; ATC GAA TAG TCT GAC TAC AAC T. 
- $\quad$ Target RNA (5'- 3'): $\beta$-actin; GCA CAA UGA AGA UCA AGA U, $\beta$-catenin; ACA UCA AGA AGG AGC UAA A, cyclin D1; ACU UCA AAU GTG UGC AGA A, c-Myc; GGA ACA AGA AGA UGA GGA A, scrambled; ATC GAA TAG TCT GAC TAC AAC T.

Fluorescence recovery of the fluorescent nanosensor in homogeneous solution. The evaluation of specificity and sensitivity of the nanosensor for detection of mRNA was performed in the presence of synthetic single stranded RNA fully complementary to PNA probe using $1 \times$ PBS solution $(\mathrm{pH} 7.4)$. Under the optimized ratio of D-GON to $\beta$-actin PNA probe $(200 \mathrm{nM})$ for fluorescence quenching ( $>95 \%)$, various concentrations $(0-200 \mathrm{nM})$ of synthetic RNA complementary to $\beta$-actin PNA probe were added. The fluorescence emission spectra (excitation wavelength $=650 \mathrm{~nm}$ ) were monitored by fluorometer SynergyMx (Biotek, UK) for 6 hours at the same time interval (every 10 minutes). The LOD was calculated based on the standard deviation $(\mathrm{SD})$ and the slope of the calibration line $(\mathrm{S})$ according to the formula; $\mathrm{LOD}=3.3(\mathrm{SD} / \mathrm{S})$.

siRNA-induced mRNA downregulation. HepG2 cells were seeded onto 6-, 96-well cell culture plate. For each transfection sample, siRNA-Lipofectamine ${ }^{\circledR} 2000$ complex was prepared according to the manufacturer's protocol. After treatment of siRNA targeting $\beta$-catenin mRNA in dose- and time- dependent manner, the mRNA expression levels of $\beta$-catenin and its target genes (cyclin D1 and c-Myc) were examined by RT-PCR and live cell fluorescence imaging.

Reverse transcription polymerase chain reaction (RT-PCR). Total RNA was isolated using TRIZOL reagent according to the manufacturer's instruction. RT-PCR was carried out as two-step reaction. Comparing the intensity of bands on agarose gel electrophoresis was accomplished using ChemiDoc MP imaging system (Bio-Rad, USA). The sequence information of primer is as follow.

- Forward primer (5'-3'): $\beta$-actin; TTC CTT CCT GGG CAT GGA GT, $\beta$-catenin; GAA ACA GCT CGT TGT ACC GC, cyclin D1; CCT GTG CTG CGA AGT GGA AA, c-Myc; CAG CGA CTC TGA GGA GGA AC. 
- Reverse primer (5'- 3'): $\beta$-actin; ACT CCT GCT TGC TGA TCC AC, $\beta$-catenin; TGA TGA GCT TGC TTT CTT GGT, cyclin D1; TCT GTT CCT CGC AGA CCT CC, c-Myc; GCT GGT GCA TTT TCG GTT GT.

Western blotting. HepG2 cells were seeded on 6-well cell culture plate and cultured with growth medium for 24 hours $\left(37^{\circ} \mathrm{C}, 5 \% \mathrm{CO} 2\right)$. According to the manufacturer's protocol, the cells were treated with siRNA (50 $\mathrm{nM}$ ) in serum-free medium using Lipofectamine ${ }^{\circledR} 2000$ for 4 hours. The transfected cells were typically collected 24 to 72 hours post-transfection and lysed for immunoblotting using Pierce RIPA buffer (Thermo Fisher Scientific, USA). Total cell lysates were separated on SDS-PAGE, transferred onto PVDF membrane (GE Healthcare, UK). The blot was probed with antibodies to $\beta$-catenin (sc-7963, Santa Cruz Biotechnology Inc., USA) and $\beta$-actin (sc-47778, Santa Cruz Biotechnology Inc., USA), and then visualized by ECL ${ }^{\text {TM }}$ Prime Western Blotting System (GE Healthcare, UK). Comparing the intensity of bands was accomplished using ChemiDoc MP imaging system (Bio-Rad, USA).

Live-cell fluorescence imaging. HepG2 cells were seeded onto Costar ${ }^{\circledR} 96$-well cell culture plate. The simply mixed solution that is be based on PNA probe and D-GON in $1 \times$ PBS solution was incubated for $30 \mathrm{~min}$ at room temperature and added with serum-free medium to a final volume of $100 \mu \mathrm{L}$. Following treatment with siRNA or small-molecule inhibitor, cells were incubated with the fluorescent nanosensor in serum-free medium for 9 hours. After briefly washing with PBS solution, the medium was replaced with fresh growth medium. Fluorescence images were recorded by IN Cell Analyzer 2000 (GE Healthcare, UK). Quantification of intracellular fluorescent signal (mean fluorescence intensity per a cell $=$ the sum of all pixel intensities forming spots / the number of cells) was obtained using multi-target analysis (MTA) software. Mean and standard deviation of triplicate values were calculated.

Scratch wound healing assay. HepG2 cells were seeded onto 12-well cell culture plate and a confluent monolayer was obtained within 24 hours. After creating a straight scratch using a pipette tip, fresh growth medium containing PNU $74654(100 \mu \mathrm{M})$ was replenished and then cells were cultured for additional 48 hours. Cell images were taken under BX71 microscope with $20 \times$ objective lens (Olympus, Japan). 
Statistical analysis. All data were obtained from at least three independent experiments. Significant differences were determined based on the Student's $t$-test where the difference were considered significant $(\mathrm{p}$ $<0.05)$. Statistical analyses were performed using Microsoft Excel 2016. 
A

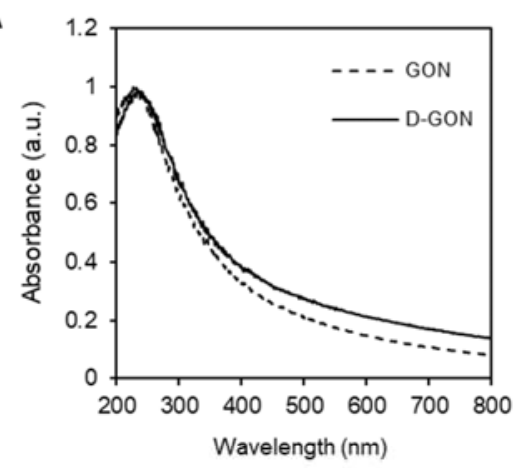

C

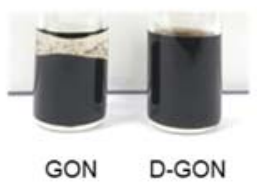

B

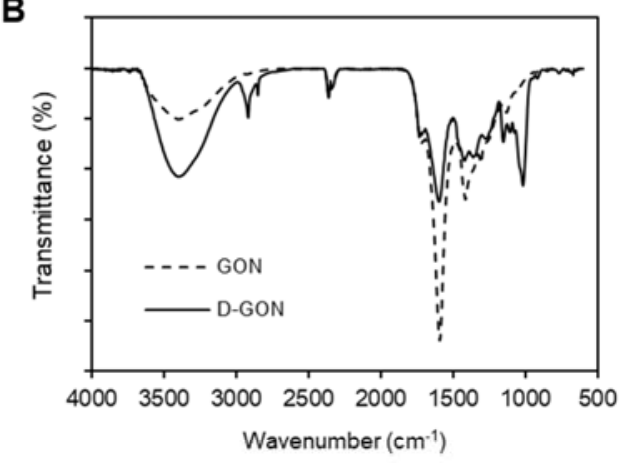

D

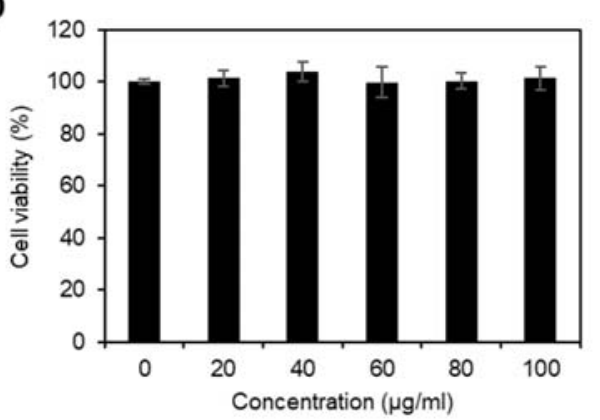

Figure S1. (A) UV-vis absorption spectra. (B) FT-IR spectra. (C) Stability test in $1 \times$ PBS solution over 24 hours. (D) Cell viability of HepG2 cells under D-GON treatment using CCK-8 assay $(n=3)$. 
A

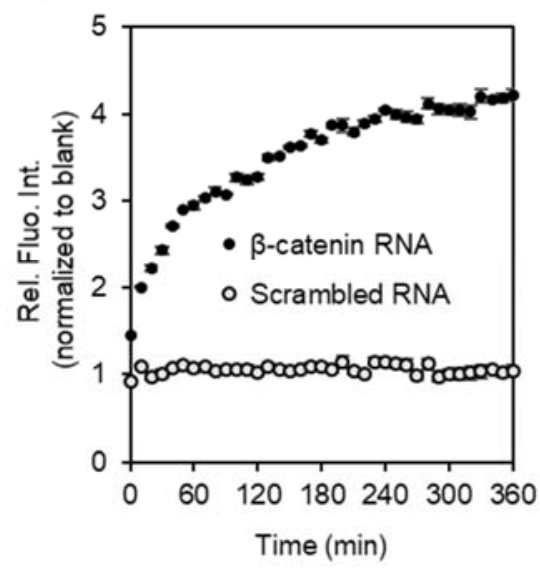

B

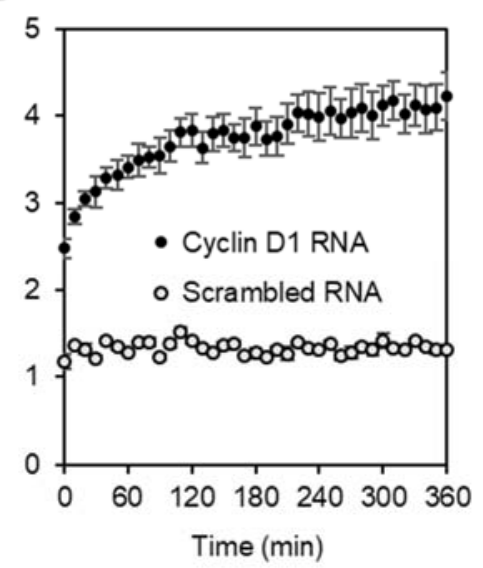

C

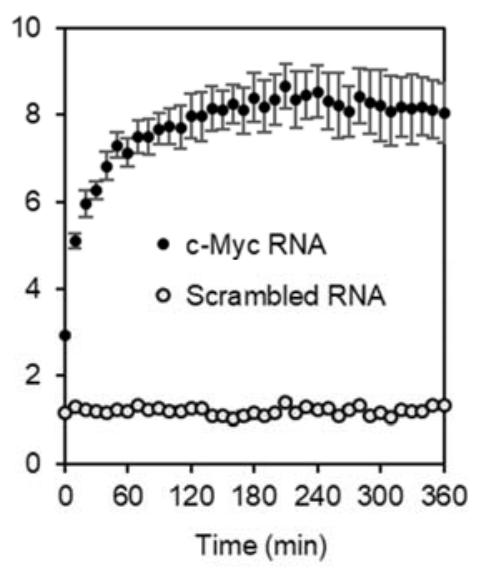

Figure S2. Time-dependent kinetics of fluorescence recovery for three PNA probes towards; (A) $\beta$-catenin, (B) cyclin D1, and (C) c-Myc mRNAs. Fluorescence emission spectra (excitation wavelength: $650 \mathrm{~nm}$, emission wavelength: $670 \mathrm{~nm}$ ) of the nanosensor in 1X PBS solution at 6 hours after adding synthetic RNAs with different sequence (200 nM). Target RNA (5'- 3'); (A) ACA UCA AGA AGG AGC UAA A, (B) ACU UCA AAU GTG UGC AGA A, (C) GGA ACA AGA AGA UGA GGA A, Scrambled RNA (5'- 3'); AGU UGU AGU CAG ACU AUU CGA U. 
A

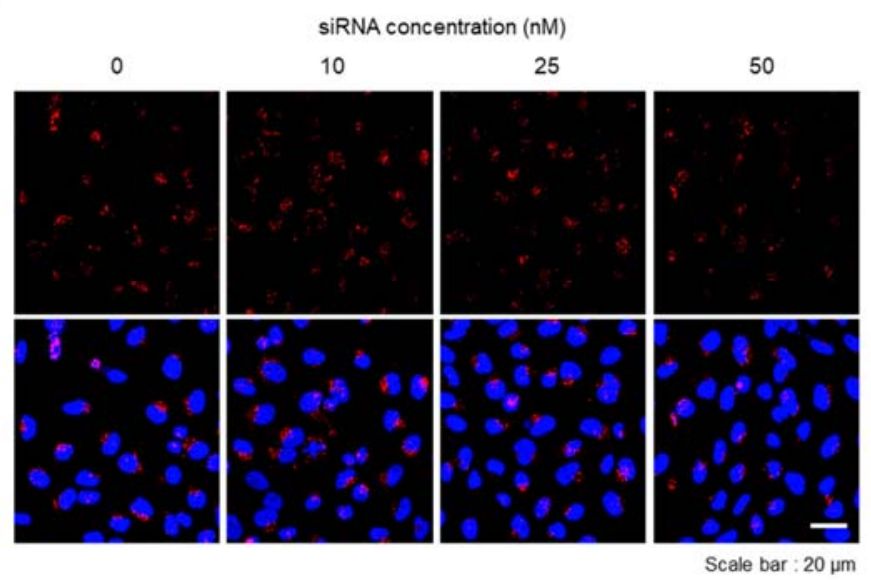

B

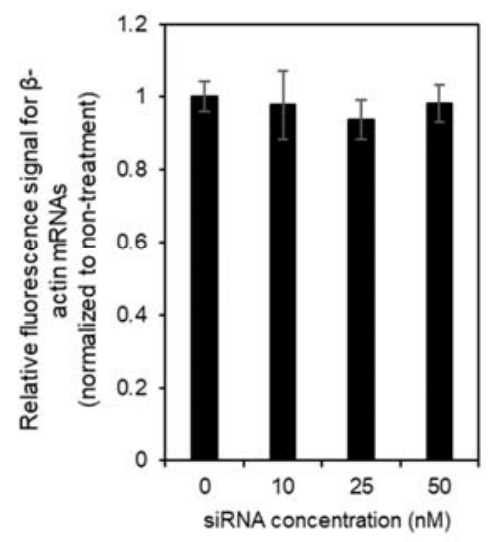

Figure S3. (A) Representative fluorescence images of $\beta$-actin mRNA in HepG2 cells after treatment of $\beta$ catenin siRNA in various concentrations. (B) Relative quantification of intracellular red signal corresponding to $\beta$-actin mRNA in the fluorescence images using MTA software $(n=3)$. 

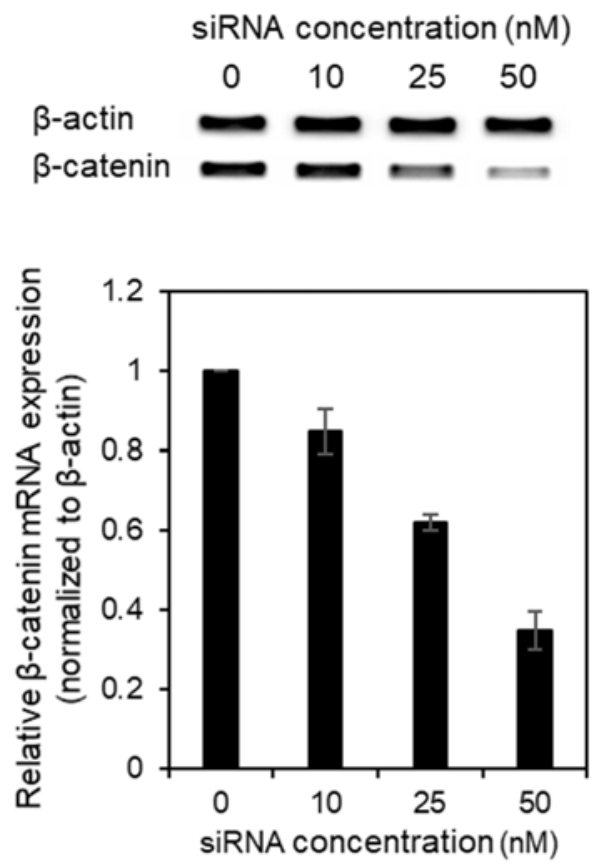

Figure S4. RT-PCR analysis in HepG2 cells after treatment of siRNA in concentration-dependent manner (n $=3)$. 


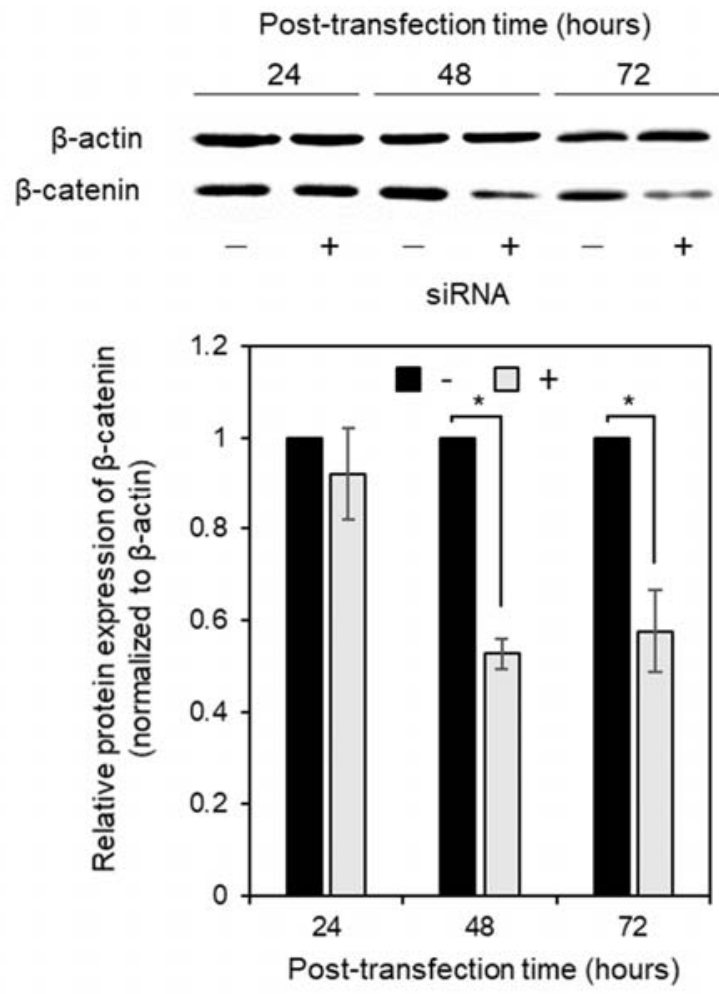

Figure S5. Western blot analysis of $\beta$-catenin protein in a time-dependent manner after $\beta$-catenin siRNA treatment (P-value was calculated by Student's t-test: * for $\mathrm{p}<.05, \mathrm{n}=3)$. 
A
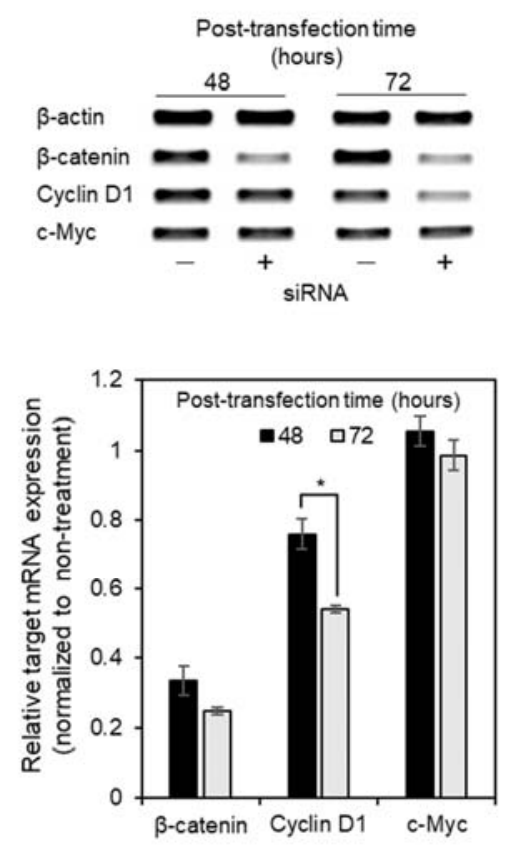

B

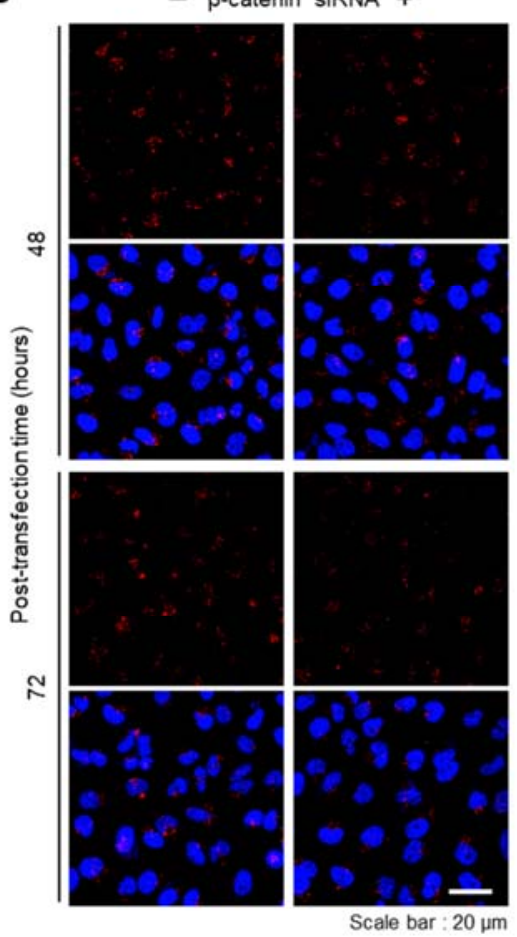

C

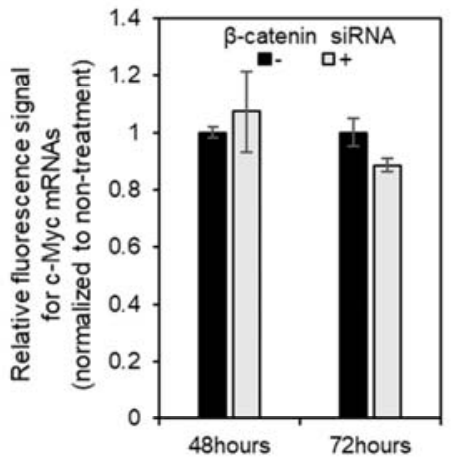

Figure S6. (A) Comparison of mRNA expression levels in a time-dependent manner following treatment with $\beta$-catenin siRNA using RT-PCR analysis (P-value was calculated by Student's t-test: * for $\mathrm{p}<.05, \mathrm{n}=$ 3). (B) Representative fluorescence images of c-Myc mRNA in HepG2 cells at 48 and 72 hours after treatment of siRNA against $\beta$-catenin mRNA. (C) Relative quantification of intracellular red signal corresponding to c-Myc mRNA using MTA software. 


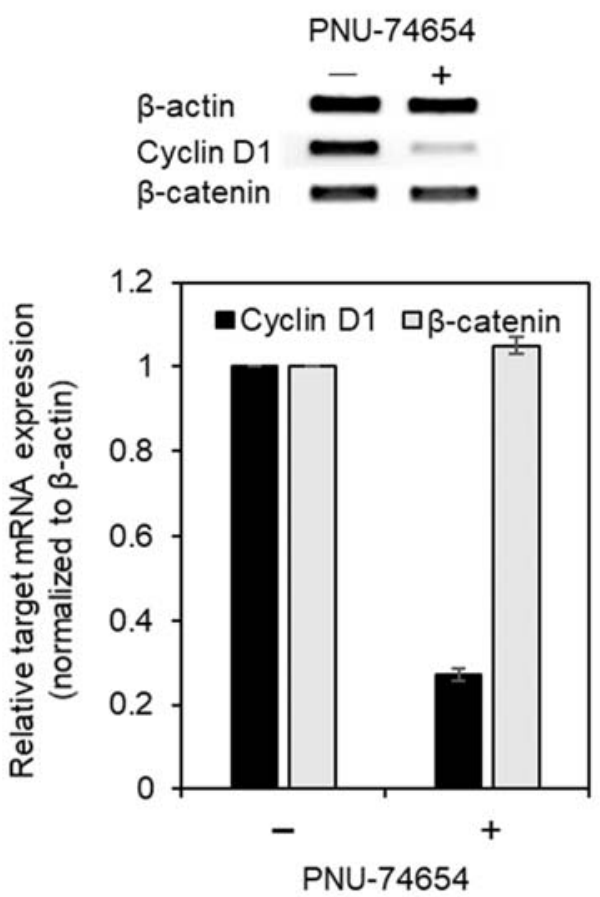

Figure S7. RT-PCR analysis in HepG2 cells treated with PNU74654 $(\mathrm{n}=3)$. 